\title{
Immunization: Must be safe or we will all be sorry
}

\author{
Greg Hammond MD FRCPC \\ Public Health Branch and Communicable Disease Control, Manitoba Health, \\ and Departments of Medical Microbiology, Infectious Diseases and Internal Medicine, \\ University of Manitoba, Winnipeg, Manitoba
}

$\mathrm{I}_{\mathrm{m}}^{\mathrm{m}}$

mmunization programs are among the most effective of all health care interventions, and they are among the few that are potentially cost-saving. However, concerns about immunization safety appear to be increasing, despite technological developments that have created safer vaccines than ever before and despite the solid scientific evidence of the safety of current immunization programs. This is due in part to the success of immunization programs, where major advances in immunization have caused the virtual disappearance of many common communicable diseases. This has resulted in the perception by some individuals that adverse events, which occasionally follow immunization, are now more common and potentially more of a hazard than the diseases for which the immunizations were developed.

Will concerns about immunization safety affect immunization programs? There is already evidence that it has. In the 1970 s, concerns about the potential side effects from pertussis vaccine in the United Kingdom resulted in diminished pertussis immunization rates and subsequent pertussis outbreaks. In 1998, public concerns in France resulted in the temporary suspension of their universal childhood hepatitis $\mathrm{B}$ immunization. Coincident to this, the universal hepatitis $\mathrm{B}$ childhood immunization program in Manitoba was challenged by an injunction to block the program (which was overturned by the court judge), but media attention negatively affected the initial uptake of the program.

A conference was held in Montreal, Quebec, from November 1 to 3,2000 , to make recommendations on immunization safety. The Immunization Subcommittee of the Public Health Working Group will bring these recommendations forward, through the Advisory Committee on Population Health, to the federal, provincial and territorial deputy ministers of health.

The discussions in Montreal centred on five areas of immunization safety, including surveillance of adverse events, immunization infrastructure such as registries, public health action, communications and immunization safety research. Over 50 participants at the meeting heard informa- tion on a wide range of issues presented at symposia, followed by group discussions.

By remarkable circumstance, during the course of the conference, an issue of immunization safety secondary to influenza vaccine emerged (1). Numerous lessons were learned from the oculorespiratory syndrome reported in association with the influenza vaccine. Postmarketing influenza vaccine surveillance detected individuals with a previously unrecognized syndrome, at a low frequency. There was effective and timely leadership, cooperation and communication to increase knowledge of this newly recognized syndrome. There was rapid mobilization of resources to acquire new information. Information was well disseminated, and epidemiological and research studies were begun.

However, immunization safety issues from this example highlighted some of the challenges to current programs, which have emerged as questions for resolution and issues for ongoing discussion.

How do we determine the clarity of roles and responsibilities for immunization safety? Do we require an arm's length, independent oversight body for reviews of concerns to the public or health professionals about immunization programs or adverse events following immunization? Who is responsible for postmarketing surveillance and analysis of adverse events? Who is responsible for the research infrastructure and research funding to determine mechanisms, frequency and distribution of adverse events? Who is responsible for the communication of adverse events? How do we establish and use technology (eg, registries) that enables record linkage studies for population-based surveillance of especially rare adverse events? Should we have a national no-fault adverse events assistance program for serious permanent sequelae after immunization? How do we assure adequate resources for the appropriate infrastructure of immunization safety?

Immunization safety requires an overarching model that supports important immunization program elements and 
assures the priority of immunization safety. This model should facilitate the establishment of protocols, procedures, management and research to assure that safe practices occur. Our deputy ministers of health will soon direct their thoughts to the recommendations for a broad range of immunization topics under review as part of the National Immunization Strategy, including vaccine procurement, program harmonization, national goals, immunization registries and immunization safety; the recommendations are being brought forward by the Public Health Working Group Subcommittee on Immunization.

Who can contribute to immunization safety? We all can. Immunization safety is a shared activity for all who participate in the planning and delivery of these important programs, along with individuals who receive vaccines. To enable this, we encourage health professionals to work together to develop, for consideration by our governments, innovative program solutions and options that assure and sustain safe immunization programs. It is also imperative that the public supports the National Immunization Strategy and these program elements. To accomplish this, the public must be a partner in an ongoing process to advise on immunization safety and to communicate concerns so that they can be addressed. We also encourage and expect our governments to work together to make available the necessary resources for the implementation of the appropriate model, programs, elements and activities.

The public deserves to be free from vaccine-preventable diseases. Technological and scientific advances will result in a dramatic increase in the number of new vaccines in the near future. Attention to issues of immunization safety is essential to maintain and enhance public and professional support for these anticipated advances in immunization and the highly effective immunization programs that are currently in place.

\section{REFERENCES}

1. Oculo-respiratory syndrome in association with the influenza vaccine: Canada, October-November 2000. Can Commun Dis Rep 2000;26:201-2. 


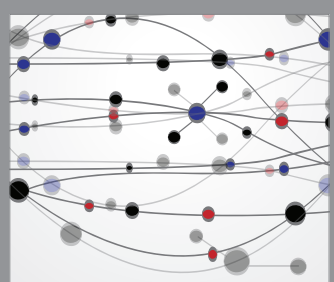

The Scientific World Journal
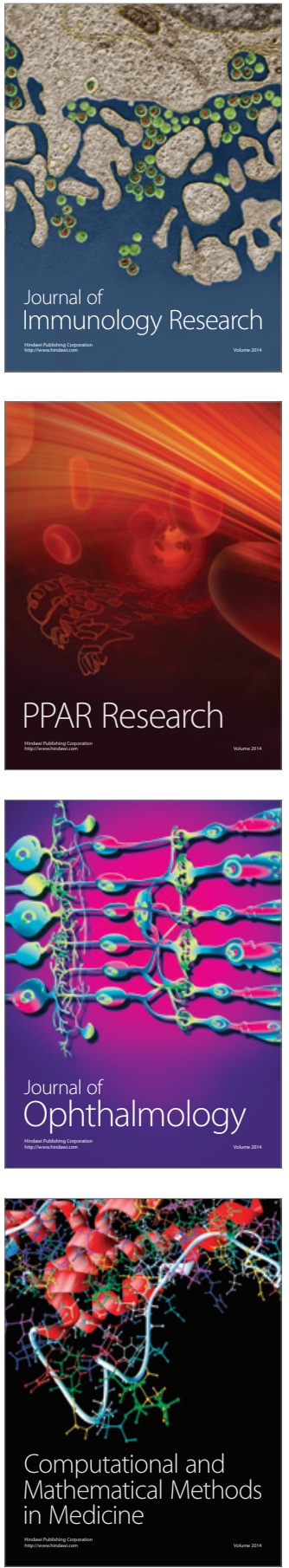

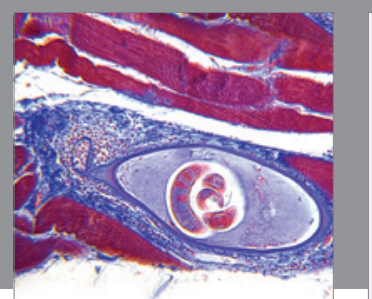

Gastroenterology Research and Practice

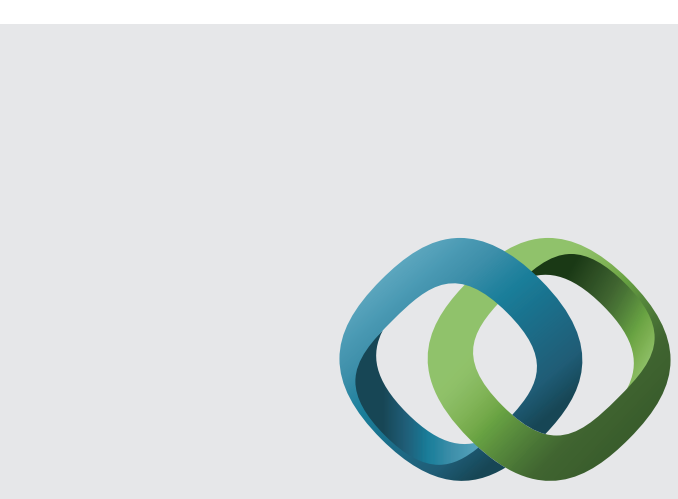

\section{Hindawi}

Submit your manuscripts at

http://www.hindawi.com
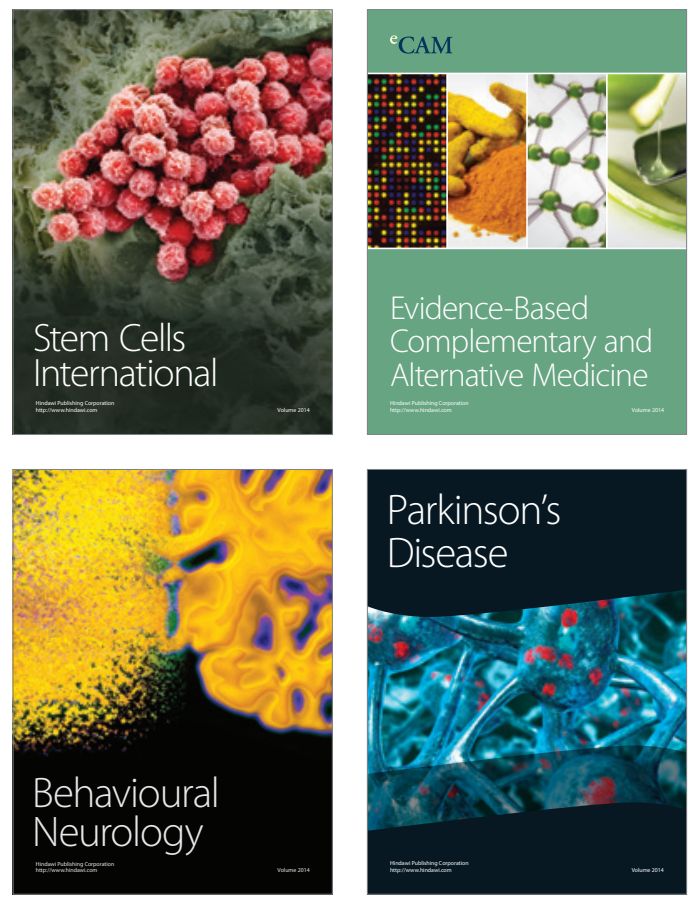
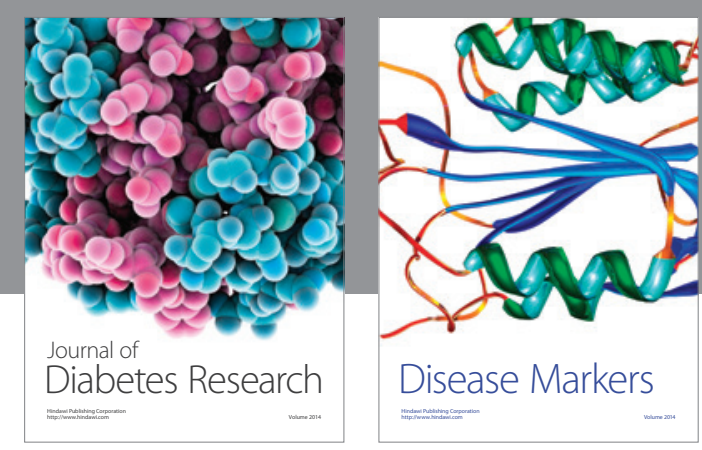

Disease Markers
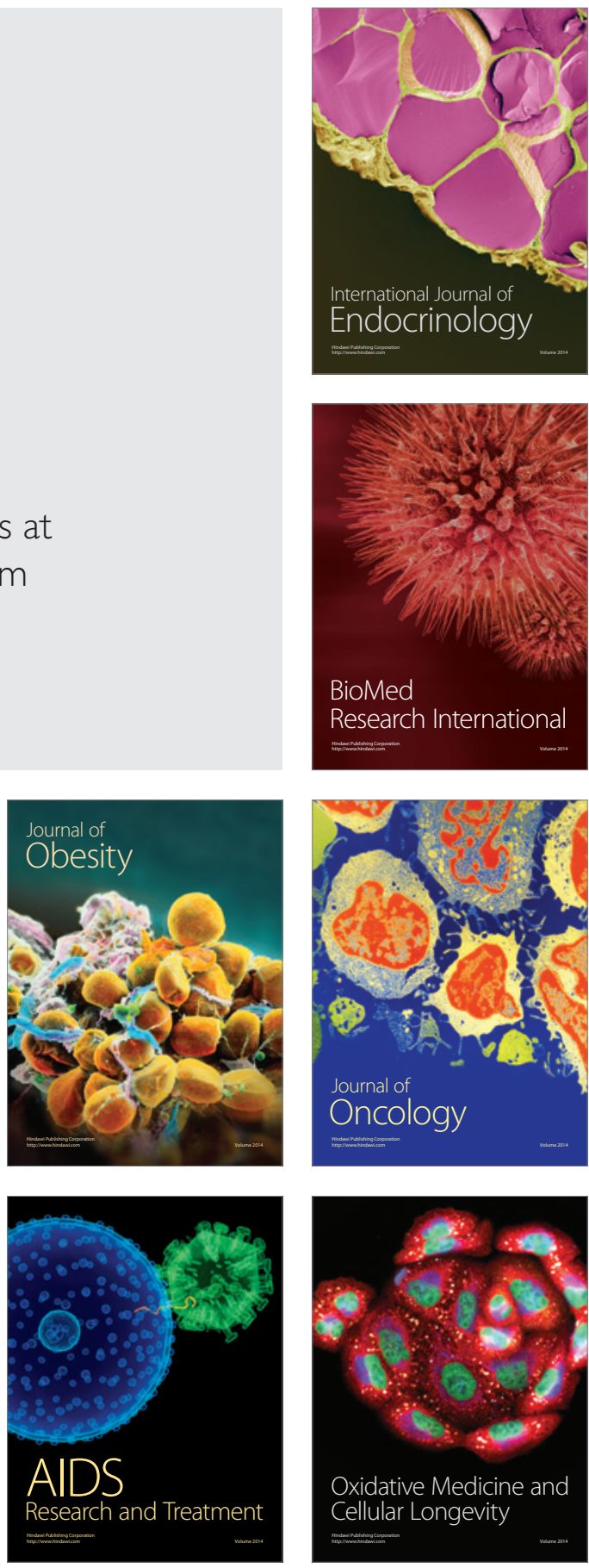